\title{
Adaptive Robust Dynamic Surface Control of Electro-hydraulic System with Unknown Nonlinear Disturbance
}

\author{
Guo Qinyang, Shi Guanglin, He Changyu, Wang Dongmei, Hu Jie \\ School of Mechanical Engineering, Shanghai Jiaotong University \\ glshi@263.net
}

\begin{abstract}
In this paper, an adaptive robust dynamic surface control strategy was proposed to improve the tracking performance of electro-hydraulic system (EHS) with unknown nonlinear disturbance and uncertain parameters. The dynamic surface control (DSC) technique was utilized to avoid the inherent "explosion of complexity" problem of the traditional back-stepping technique. The proposed control method could not only simplify the design process of the robust controller, but also estimate the uncertain parameters in combination with discontinuous projection operator. Based on Lyapunov stability theory, all signals of the closed-loop system were certified to be bounded, and semi-global asymptotic stability of the system was obtained. The simulation results verified that the control strategy is of anti-interference, and can enhance the trajectory tracking accuracy of the electro-hydraulic trajectory tracking system.
\end{abstract}

Keywords: electro-hydraulic system; uncertain parameters; nonlinear disturbance; dynamic surface control; adaptive robust control

\section{Introduction}

Electro-hydraulic systems (EHS) especially valve controlled asymmetric-cylinder systems (VCCS) have been widely used in industry on account of the advantages of small size-to-power ratios, fast response and ability to provide large force; examples like forging equipments, shield tunnelling machines, vehicle active suspensions, hydraulic load simulators, bionic robots, and so on[1-4]. However, affected by dead zone and volume flow nonlinearity of the control valve, internal leakage and volume flow unbalance of the asymmetric cylinder, and so on, the dynamic behaviour of the VCCS is highly nonlinear[5]. Due to the friction of the hydraulic components, the physical characteristics of the hydraulic oil vary with the working environment, many parameters in the VCCS are uncertain[6,7]. For these reasons, the development of the highperformance closed-loop controllers becomes more difficult.

To meet the increasingly rigid performance demands in high accuracy trajectory tracking of the EHS, advanced control techniques especially nonlinear control schemes have received highly respected. Kwanchai and Issaree designed a hybrid of fuzzy and fuzzy self-tuning PID controller for EHS to adjust the velocity of the hydraulic motor[8]. However, it is undeniable that PID control is increasing overwhelmed by high performance demands in modern industry[9], especially for those VCCS with highly nonlinear behaviours and modelling uncertainties. By using sliding model control with adaptive fuzzy compensation, the nonlinear problem caused by the proportional valve dead zone was solved in [10]. Nevertheless, aforementioned nonlinear control strategy relies on precise modelling of the considered system, and the potential uncertain parameters, unknown nonlinearity, and unknown external disturbance in practical VCCS may severely exacerbate the control performance, even instability may occur. An adaptive robust backstepping strategy was designed in [11] to tackle the unknown nonlinear parameters of the VCCS, and the superior performance was obtained. However, the research of the adaptive backstepping controllers requires analytic calculation of the partial derivatives of certain stabilizing functions, and inevitably suffer the "explosion of complexity" problem[12]. It is worth noting that, as the order of the nonlinear system increases, analytic calculation of the derivatives becomes prohibitive[13].

In this paper, by fully considering parametric uncertainties, unknown nonlinear disturbance and the "explosion of complexity" problem, an adaptive robust dynamic surface control method was designed for high performance tracking control of VCCS. By employing Robust DSC technique, the inherent "explosion of complexity" problem of the traditional backstepping method was avoided, and the effect of the unknown nonlinear disturbance was suppressed. Combining the discontinuous projection operator[14], the adaptive law of the VCCS uncertain parameters was designed. The stability 
analysis of the closed-loop system was accomplished. At last, the comparative simulation results were obtained to verify the high tracking performance of the present controller for the VCCS.

\section{Problem Formulation and Mathematical Model of VCCS}

The considered VCCS is shown in Fig.1. An electro-hydraulic hybrid driven gait simulator was developed for in vitro studies of the foot biomechanics, and the VCCS was used to extend the workspace of the parallel mechanism along the fore-aft direction of the gait. The tibia of the cadaveric foot was fixed on the moving platform of the parallel mechanism, and the motion of the tibia was synergistically recreated by the parallel mechanism and the VCCS. The main objective in this paper is to control the cylinder with unknown nonlinear disturbance to a designated trajectory as closely as possible.

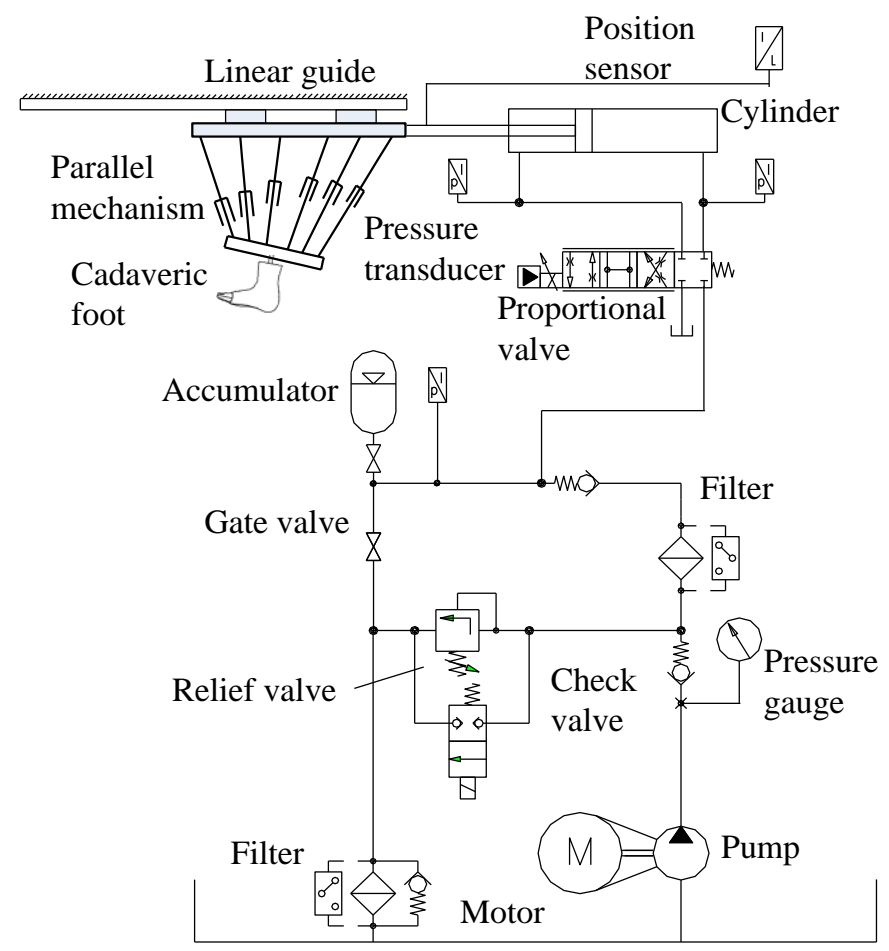

Fig. 1: The architecture of the considered hydraulic system.

Firstly, some reasonable assumptions can be considered to facilitate the mathematical modelling process of the VCCS[11]: (1) The supply pressure of the pump station can be considered to be unchanged. (2) The proportional valve has an ideal symmetrical spool and a zero overlap window. (3) The radial clearance leakage of the proportional valve and the external leakage of the EHS can be neglected. (4) Since a high-response proportional valve was used, the control applied to the proportional valve can be considered proportional to the spool position. And the fluid dynamics of the asymmetric cylinder can be written as follows,

$$
\left\{\begin{array}{l}
\frac{V_{1}}{\beta_{e}} \dot{p}_{1}=-A_{1} \dot{x}_{c}-C_{i}\left(p_{1}-p_{2}\right)-C_{e 1} p_{1}+q_{1} \\
\frac{V_{2}}{\beta_{e}} \dot{p}_{2}=A_{2} \dot{x}_{c}+C_{i}\left(p_{1}-p_{2}\right)-C_{e 2} p_{2}-q_{2}
\end{array}\right.
$$

where $x_{c}$ is the displacement of the cylinder, $V_{1}=V_{01}+A_{1} x_{c}$ and $V_{2}=V_{02}-A_{2} x_{c}$ are the effectively control volume of the non-rod chamber and the rod chamber of the cylinder respectively, $V_{01}$ and $V_{02}$ are the original control volumes of the two chambers respectively, $A_{1}$ and $A_{2}$ are the effective cross-section area of the two sides of the piston respectively, $\beta_{e}$ is the 
effective oil buck modulus of the working fluid, $p_{1}$ and $p_{2}$ are the pressures at the non-rod port and the rod port of the cylinder. $C_{i}$ denotes the internal leakage coefficient of the cylinder, $C_{e 1} \approx 0$ and $C_{e 2} \approx 0$ are the external leakage coefficients of the cylinder, $q_{1}$ and $q_{2}$ denotes the flow rates of the two chambers respectively. Considering the proportional valve mentioned above, the flow rates $q_{1}$ and $q_{2}$ can be described as

$$
\begin{gathered}
\left\{\begin{array}{c}
q_{1}=s(u) k_{q} u \sqrt{p_{s}-p_{1}}+s(-u) k_{q} u \sqrt{p_{1}-p_{r}} \\
q_{2}=s(u) k_{q} u \sqrt{p_{2}-p_{r}}+s(-u) k_{q} u \sqrt{p_{s}-p_{2}}
\end{array}\right. \\
\mathrm{s}(*)= \begin{cases}1 & * \geq 0 \\
0 & *<0\end{cases} \\
k_{q}=C_{d} w \sqrt{2 / \rho}
\end{gathered}
$$

where $C_{d}$ and $w$ are the flow coefficient and area gradient of the proportional valve respectively, $\rho$ is the density of the hydraulic oil, $p_{s}$ and $p_{r}=0$ denote the supply pressure and the tank pressure respectively. And the dynamic equation governing the motion of the hydraulic cylinder can be written as

$$
A_{1} p_{1}-A_{2} p_{2}=m \ddot{x}_{c}+b \dot{x}_{c}+f(t)
$$

where $m$ denotes the equivalent mass of the load including the piston rod, $b$ is the viscous friction coefficient of the VCCS, $f(t)$ denotes the unknown nonlinear disturbance including nonlinear friction, the reaction of the parallel mechanism, and the reaction of the ground against the soles of the feet during the gait simulation.

Synthesizing (1)-(5), the state variables can be defined as $\mathbf{x}=\left[x_{1}, x_{2}, x_{3}\right]^{T}=\left[x_{c}, \dot{x}_{c}, A_{1} p_{1}-A_{2} p_{2}\right]^{T}$.Generally speaking, the accuracy values of $\beta_{e}, m, b, C_{i}, k_{q}, A_{1}$ and $A_{2}$ are difficult to obtain. In order to simplify the state-space equation, the unknown parameter set $\boldsymbol{\theta}=\left[\theta_{1}, \theta_{2}, \theta_{3}, \theta_{4}, \theta_{5}, \theta_{6}\right]^{\mathrm{T}}$ is defined, where $\theta_{1}=1 / m, \theta_{2}=b / m, \theta_{3}=\beta_{e} A_{1}, \theta_{4}=\beta_{e} C_{i}, \theta_{5}=\beta_{e} k_{q}$ and $\theta_{6}=\beta_{e} A_{2}$. Then the state-space function of the VCCS can be written as

$$
\left\{\begin{array}{l}
\dot{x}_{1}=x_{2} \\
\dot{x}_{2}=\theta_{1} x_{3}-\theta_{2} x_{2}+d \\
\dot{x}_{3}=-\left(h_{1} \theta_{3}+h_{2} \theta_{6}\right) x_{2}-\left(h_{1}+h_{2}\right) \theta_{4}\left(p_{1}-p_{2}\right)+\left(h_{1} g_{1}+h_{2} g_{2}\right) \theta_{5} u \\
y=x_{1}
\end{array}\right.
$$

where $d=-f(t) / m, g_{1}=s(u) \sqrt{p_{s}-p_{1}}+s(-u) \sqrt{p_{1}}, g_{2}=s(u) \sqrt{p_{2}}+s(-u) \sqrt{p_{s}-p_{2}}, h_{1}=A_{1} /\left(V_{01}+A_{1} x_{1}\right)$, and $h_{2}=A_{2} /\left(V_{02}-A_{2} x_{1}\right)$.

Related to adaptive controller designing, the following assumptions are required.

Assumption 1: During the gait simulation, the desired trajectory of the hydraulic cylinder $x_{d}$ and its derivatives $\dot{x}_{d}$ are bounded, continuous, and known.

Assumption 2: The unknown parameter set $\boldsymbol{\theta}$ and the unknown nonlinear disturbance $d$ satisfy that

$$
\boldsymbol{\theta} \in \Omega_{\boldsymbol{\theta}} \triangleq\left\{\boldsymbol{\theta}: \boldsymbol{\theta}_{\min } \leq \boldsymbol{\theta} \leq \boldsymbol{\theta}_{\max }\right\}
$$




$$
|d(t, x)| \leq D
$$

where $\boldsymbol{\theta}_{\text {min }}=\left[\theta_{1 \text { min }}, \theta_{2 \min }, \theta_{3 \min }, \theta_{4 \min }, \theta_{5 \min }, \theta_{6 \min }\right]^{\mathrm{T}}, \boldsymbol{\theta}_{\text {max }}=\left[\theta_{1 \max }, \theta_{2 \max }, \theta_{3 \max }, \theta_{4 \max }, \theta_{5 \max }, \theta_{6 \max }\right]^{\mathrm{T}}$ are known, $D$ is a known positive constant. In this paper, $\hat{*}$ denotes the estimation of $*$, and $\tilde{*}=*-\hat{*}$ denotes the estimation error.

\section{Adaptive Robust Dynamic Surface Controller Design}

\subsection{Controller Design}

An improved DSC technique would be employed to construct the composite controller for the VCCS, and the "explosion of complexity" problem of the traditional backstepping method can be avoided.

Choose the tracking error of the cylinder as the first error surface, seeing equation (9), and the first virtual controller $x_{2 d}$ has been given in equation (10),

$$
\begin{gathered}
s_{1}=x_{1}-x_{d} \\
x_{2 d}=\dot{x}_{d}-k_{1} s_{1}
\end{gathered}
$$

where $k_{1}>0$ is the first feedback gain. A first order filter $x_{2 f}$ can be introduced to avoid the analytic differentiation of the virtual controller $x_{2 d}$, and let the virtual controller $x_{2 d}$ pass through the first order filter, yields

$$
\tau_{2} \dot{x}_{2 f}+x_{2 f}=x_{2 d} ; \quad x_{2 f}(0)=x_{2 d}(0)
$$

where $\tau_{2}$ is the time constant of the filter. Then the second error surface $s_{2}$ and the filtered error $y_{2}$ can be defined as,

$$
\begin{aligned}
& s_{2}=x_{2}-x_{2 f} \\
& y_{2}=x_{2 f}-x_{2 d}
\end{aligned}
$$

Considering the uncertain parameter and the unknown nonlinear disturbance of the VCCS (6), a robust adaptive method is utilized to design the second virtual controller,

$$
\begin{aligned}
& x_{3 d}=x_{3 d}^{a}+x_{3 d}^{s} ; x_{3 d}^{a}=\left(\hat{\theta}_{2} x_{2}+\dot{x}_{2 f}\right) / \hat{\theta}_{1} ; \\
& x_{3 d}^{s}=x_{3 d}^{s 1}+x_{3 d}^{s 2} ; x_{3 d}^{s 1}=-k_{2} s_{2} / \theta_{1 \min }
\end{aligned}
$$

where $k_{2}>0$ is the second feedback gain. $x_{3 d}^{a}$ is an adjustable model-based feed-forward control law which can be used to obtain an improved model compensation via parameter adaptation. $x_{3 d}^{s}$ is a nonlinear robust feedback law to stabilize the nominal model of the system, such that the effect of the unknown nonlinear disturbance $d$ can be attenuated. Furthermore, another first order filter $x_{3 f}$ can be introduced to avoid analytic differentiation of the virtual controller $x_{3 d}$, let the virtual controller $x_{3 d}$ pass through the first order filter, yields

$$
\tau_{3} \dot{x}_{3 f}+x_{3 f}=x_{3 d} ; \quad x_{3 f}(0)=x_{3 d}(0)
$$

where $\tau_{3}$ is the time constant of the filter. And the third error surface $s_{3}$, and the filtered error $y_{3}$ can be written as 


$$
\begin{aligned}
& s_{3}=x_{3}-x_{3 f} \\
& y_{3}=x_{3 f}-x_{3 d}
\end{aligned}
$$

Combining equations (12), (13) and (14), the time derivative of $s_{2}$ can be obtained

$$
\dot{s}_{2}=\theta_{1}\left(s_{3}+y_{3}+x_{3 d}^{s 2}\right)-\theta_{1} k_{2} s_{2} / \theta_{1 \min }+\tilde{\theta}_{1} x_{3 d}^{a}-\tilde{\theta}_{2} x_{2}+d
$$

Defining a Semi-definite Lyapunov equation $V_{2}=s_{2}^{2} / 2$, the time derivative of $V_{2}$ can be written as

$$
\dot{V}_{2}=s_{2} \theta_{1}\left(s_{3}+y_{3}+x_{3 d}^{s 2}\right)-\theta_{1} k_{2} s_{2}^{2} / \theta_{1 \min }+s_{2}\left(\tilde{\theta}_{1} x_{3 d}^{a}-\tilde{\theta}_{2} x_{2}+d\right)
$$

Choosing the appropriate robust control law $x_{3 d}^{s 2}$ to satisfy $s_{2} x_{3 d}^{s 2} \leq 0$ and $s_{2} d \leq \delta_{1}$, and the control input can be designed as

$$
\begin{aligned}
& u=u_{a}+u_{s} ; u_{s}=u_{s 1}+u_{s 2} ; u_{s 1}=-k_{3} s_{3} / \theta_{5 \min } ; \\
& u_{a}=\frac{\left(h_{1} \hat{\theta}_{3}+h_{2} \hat{\theta}_{6}\right) x_{2}+\left(h_{1}+h_{2}\right) \hat{\theta}_{4}\left(x_{31}-x_{32}\right)+\dot{x}_{3 f}}{\hat{\theta}_{5}\left(h_{1} g_{1}+h_{2} g_{2}\right)}
\end{aligned}
$$

where $k_{3}>0$ is the third feedback gain. Synthesizing VCCS (6), equations (16), and (17), the time derivative of $s_{3}$ can be obtained.

$$
\begin{aligned}
\dot{s}_{3}= & -\theta_{5}\left(h_{1} g_{1}+h_{2} g_{2}\right) k_{3} s_{3} / \theta_{5 \min }+\theta_{5}\left(h_{1} g_{1}+h_{2} g_{2}\right) u_{s 2}-h_{1} \tilde{\theta}_{3} x_{2} \\
& -\left(h_{1}+h_{2}\right) \tilde{\theta}_{4}\left(x_{31}-x_{32}\right)+\tilde{\theta}_{5}\left(h_{1} g_{1}+h_{2} g_{2}\right) u_{a}-h_{2} \tilde{\theta}_{6} x_{2}
\end{aligned}
$$

Defining a Semi-definite Lyapunov equation $V_{3}=s_{3}^{2} / 2$, and the time derivative of $V_{2}$ can be written as

$$
\begin{aligned}
\dot{V}_{3}= & -\theta_{5}\left(h_{1} g_{1}+h_{2} g_{2}\right) k_{3} s_{3}^{2} / \theta_{5 \min } \\
& -s_{3}\left(h_{1} \tilde{\theta}_{3}+h_{2} \tilde{\theta}_{6}\right) x_{2}-s_{3}\left(h_{1}+h_{2}\right) \tilde{\theta}_{4}\left(x_{31}-x_{32}\right) \\
& +s_{3} \theta_{5}\left(h_{1} g_{1}+h_{2} g_{2}\right) u_{s 2}+s_{3} \tilde{\theta}_{5}\left(h_{1} g_{1}+h_{2} g_{2}\right) u_{a}
\end{aligned}
$$

Choosing the appropriate control input $u_{s 2}$ to satisfy $s_{3} u_{s 2} \leq 0$. Based on discontinuous projection operator[15], The adaptive law is given by

$$
\dot{\hat{\boldsymbol{\theta}}}=\operatorname{Proj}(\Gamma \boldsymbol{\pi})
$$

where $\Gamma>0$ is a diagonal adaptation rate matrix and $\pi$ is an adaption function based on the designed controller which can be synthesized as

$$
\boldsymbol{\pi}=\left[x_{3 d}^{a} s_{2},-x_{2} s_{2},-h_{1} x_{2} s_{3},-\left(h_{1}+h_{2}\right)\left(p_{1}-p_{2}\right) s_{3},\left(h_{1} g_{1}+h_{2} g_{2}\right) u_{a} s_{3},-h_{2} s_{3}\right]^{T}
$$

and the discontinuous projection can be written as 


$$
\operatorname{Proj}_{\hat{\theta}}(\bullet)=\left\{\begin{array}{cc}
0 & \text { if } \hat{\theta}_{i}=\theta_{i \max } \text { and } \bullet>0 \\
0 & \text { if } \hat{\theta}_{i}=\theta_{i \min } \text { and } \bullet<0 \\
\bullet & \text { otherwise }
\end{array}\right.
$$

Consequently, for any adaptive function $\pi$, the projection mapping (25) guarantees

$$
\left\{\begin{array}{l}
\theta_{i \min } \leq \hat{\theta}_{i} \leq \theta_{i \max } \\
\tilde{\boldsymbol{\theta}}^{T}\left(\boldsymbol{\pi}-\Gamma^{-1} \operatorname{Proj}_{\hat{\boldsymbol{\theta}}}(\Gamma \boldsymbol{\pi})\right) \leq 0
\end{array}(i=1,2 \cdots 6)\right.
$$

\subsection{Stability Analysis}

Based on Assumption 1, Assumption 2, and the designed controller in section 3.1, a Lyapounov function as shown in equation (27) candidates of the whole closed-loop system is employed to analysis the states of the VCCS.

$$
V=V_{2}+V_{3}+\frac{1}{2} \sum_{i=2}^{3} y_{i}^{2}+\frac{1}{2} \tilde{\boldsymbol{\theta}}^{T} \Gamma^{-1} \tilde{\boldsymbol{\theta}}
$$

Using Young's inequalities and considering the following facts

$$
\begin{gathered}
y_{2} \dot{y}_{2} \leq-\frac{y_{2}^{2}}{\tau_{2}}+M_{2}\left|y_{2}\right| \leq-\frac{y_{2}^{2}}{\tau_{2}}+y_{2}^{2}+\frac{1}{4} M_{2}^{2} \\
y_{3} \dot{y}_{3} \leq-\frac{y_{3}^{2}}{\tau_{3}}+M_{3}\left|y_{3}\right| \leq-\frac{y_{3}^{2}}{\tau_{3}}+y_{3}^{2}+\frac{1}{4} M_{3}^{2} \\
s_{2} s_{3} \leq s_{2}^{2}+\frac{s_{3}^{2}}{4} \\
s_{2} y_{2} \leq s_{2}^{2}+\frac{y_{2}^{2}}{4}
\end{gathered}
$$

where $M_{2}=\left|-\dot{x}_{2 d}\right|, M_{3}=\left|-\dot{x}_{3 d}\right|$. Combining equations (27)-(31), the time derivative of $V$ can be written as

$$
\begin{aligned}
\dot{V} \leq & -\theta_{1}\left(\frac{k_{2} \theta_{1}}{\theta_{1 \min }}-2\right) s_{2}^{2}-\left(\frac{k_{3} \theta_{5}}{\theta_{5 \min }}\left(h_{1} g_{1}+h_{2} g_{2}\right)-\frac{\theta_{1}}{4}\right) s_{3}^{2} \\
& -\left(\frac{1}{\tau_{2}}-\frac{\theta_{1}}{4}-1\right) y_{2}^{2}-\left(\frac{1}{\tau_{3}}-1\right) y_{3}^{2}+\tilde{\boldsymbol{\theta}}^{T} \boldsymbol{\pi} \\
& +\frac{M_{2}^{2}}{4}+\frac{M_{3}^{2}}{4}-\tilde{\boldsymbol{\theta}}^{T} \Gamma^{-1} \dot{\hat{\boldsymbol{\theta}}}+\delta_{1}^{2}+\delta_{2}^{2}
\end{aligned}
$$

Reasonably select the control parameters and meet the following conditions, 


$$
\left(k_{2}-2\right) \theta_{1} \geq a ; \frac{1}{\tau_{3}}-1 \geq a ; k_{3}\left(h_{1} g_{1}+h_{2} g_{2}\right)-\frac{\theta_{1}}{4} \geq a ; \frac{1}{\tau_{2}}-\frac{\theta_{1}}{4}-1 \geq a ; \exists a>0
$$

Combining equation (26), yields

$$
\dot{V} \leq-2 a V+C
$$

where $C=\frac{M_{2}^{2}}{4}+\frac{M_{3}^{2}}{4}+\delta_{1}^{2}+\delta_{2}^{2}+a \tilde{\theta}^{T} \Gamma^{-1} \tilde{\theta}$. According to [16], if $V \geq \mu, \mu$ is a positive constant. Choosing $a \geq C /(2 \mu)$, then $\dot{V} \leq 0$ can be guaranteed. Thus, all signals of the closed-loop control system are uniformly ultimately bounded.

\section{Comparative Simulation Results}

In this section, Matlab/Simulink was utilized to verify the effectiveness of the proposed control strategy. S-function and function block were used to construct the VCCS model and the proposed controller respectively. For the simulink, the solver was set to be fixed step ode3, the step size was set to be $1 \times 10^{-3} \mathrm{~s}$. The control block is illustrated in Figure 2 , and the parameters of the considered VCCS are given in Table 1.

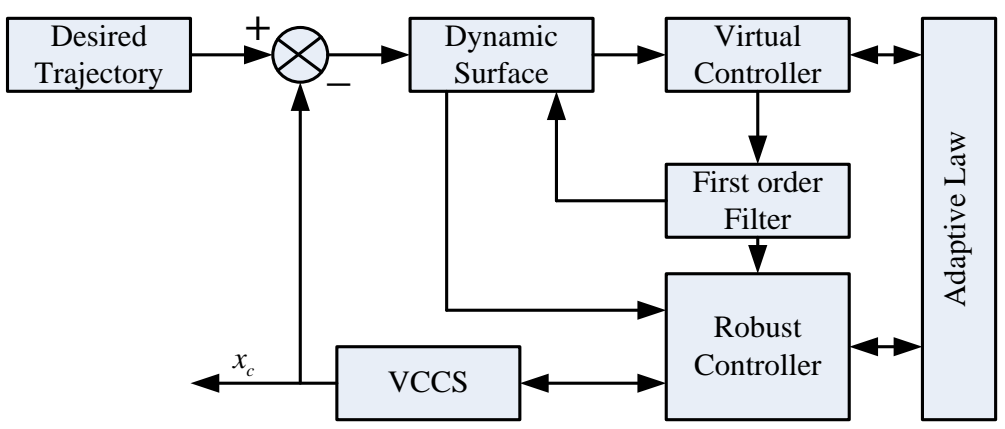

Fig. 2: Control block of the EHS.

Table 1: Parameters of the EHS.

\begin{tabular}{|l|l|l|l|}
\hline Parameter & Value & Parameter & Value \\
\hline$p_{s}$ & $9 \times 10^{6} \mathrm{~Pa}$ & $C_{i}$ & $10^{-15} \mathrm{~m}^{3} \mathrm{~s}^{-1} / \mathrm{Pa}$ \\
\hline$m$ & $600 \mathrm{~kg}$ & $k_{q}$ & $1.019 \times 10^{-7}$ \\
\hline$A_{1}$ & $3.117 \times 10^{-3} \mathrm{~m}^{2}$ & $b$ & $3150 \mathrm{~N} \cdot \mathrm{s} / \mathrm{m}$ \\
\hline$A_{2}$ & $1.527 \times 10^{-3} \mathrm{~m}^{2}$ & $V_{01}$ & $0.8 \times 10^{-3} \mathrm{~m}^{3}$ \\
\hline$\beta_{e}$ & $200 \times 10^{6} \mathrm{~Pa}$ & $V_{02}$ & $1.88 \times 10^{-3} \mathrm{~m}^{3}$ \\
\hline
\end{tabular}

The initial values of the uncertain parameters were set to be $\theta_{10}=0.0017, \theta_{20}=5.25, \theta_{30}=6.2345 \times 10^{5}, \theta_{40}=2 \times 10^{-7}$, $\theta_{50}=20.3791$, and $\theta_{60}=3.0536 \times 10^{5}$. While the bounds of the uncertain parameters were set to be $\theta_{1 \min }=0.0015$, $\theta_{1 \min }=0.0015, \theta_{2 \min }=5.15, \theta_{3 \min }=6.23 \times 10^{5}, \theta_{4 \min }=1.5 \times 10^{-7}, \theta_{5 \min }=20.37, \theta_{6 \min }=3.05 \times 10^{5} ; \theta_{1 \max }=0.002, \theta_{2 \max }=5.35$, $\theta_{3 \max }=6.24 \times 10^{5}, \theta_{4 \max }=2.5 \times 10^{-7}, \theta_{5 \max }=20.39$, and $\theta_{6 \max }=3.06 \times 10^{5}$.

To investigate the effectiveness and high performance of the proposed adaptive robust dynamic surface controller, the following simulation cases are given: case 1: control performance comparison between traditional proportion-integralderivative controller (PID) and traditional robust controller (RC) without unknown nonlinear disturbance; case 2: control performance comparison of PID, RC and the proposed adaptive robust dynamic surface controller (ARDSC). And the desired trajectory and the unknown nonlinear disturbance are given by 


$$
\begin{aligned}
& x_{d}=0.3 \sin (0.4 \pi \mathrm{t}) \quad(\mathrm{m}) \\
& f(t)=60 \sin (0.8 \pi t) \quad(\mathrm{N})
\end{aligned}
$$

The simulation results of case 1 are illustrated in Fig.3. The tracking error of the PID controller was about $-10 \mathrm{~mm} 3$ $\mathrm{mm}$, and that of the RC was about $-0.1 \mathrm{~mm} \sim 0.4 \mathrm{~mm}$. The tracking error of the RC was significantly reduced, and the hysteresis phenomenon was almost be eliminated. It can be found that the control performance of the RC is much better than that of traditional PID controller. The tracking results and the parameter estimation results of case 2 are illustrated in Fig.4. Due to the effects of the unknown external disturbance, the maximum tracking error of the PID controller was about $25 \mathrm{~mm}$, simultaneously, the tracking error of the RC was about $-0.8 \mathrm{~mm} \sim 0.6 \mathrm{~mm}$, and that of the ARDSC was about -0.5 $\mathrm{mm} \sim 0.4 \mathrm{~mm}$. The simulation results illustrate an excellent effectiveness of the proposed ARDSC method, especially for those VCCS with uncertain parameters and unknown external disturbance.

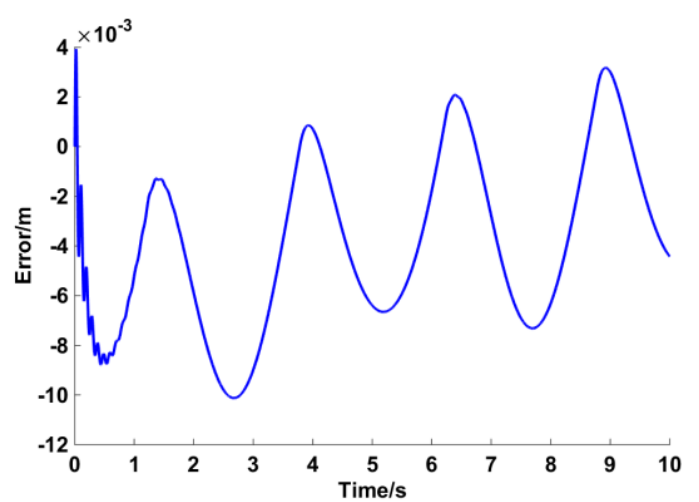

(a) PID

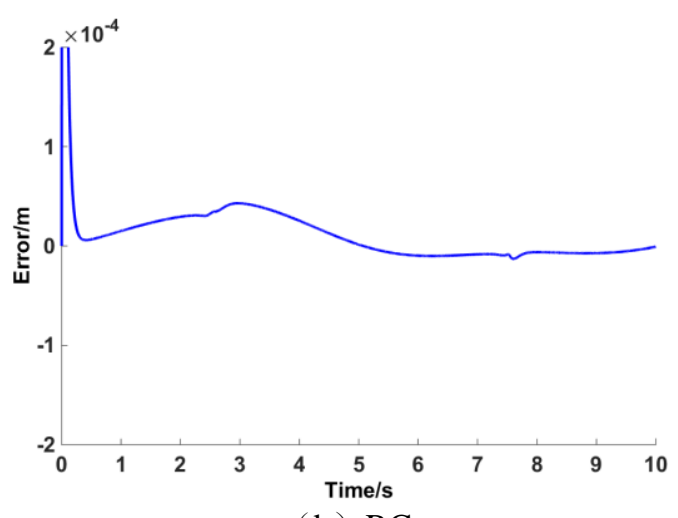

(b) $\mathrm{RC}$

Fig. 3: Comparative tracking performance of case 1.

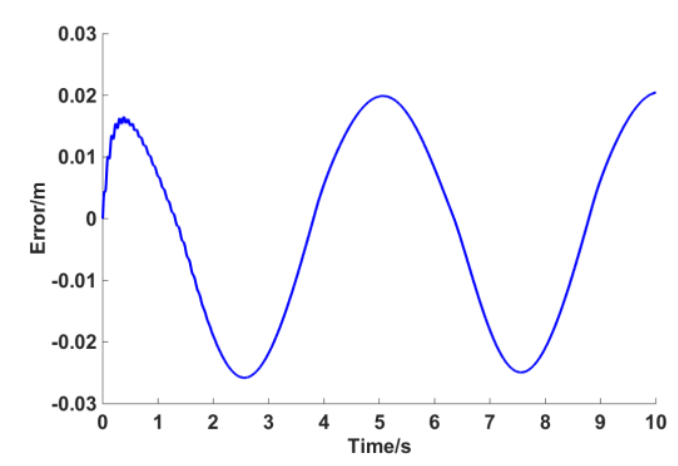

(a) PID

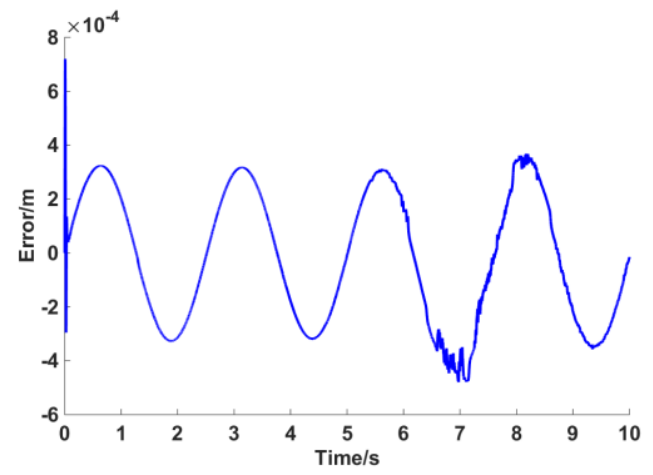

(c) ARDSC

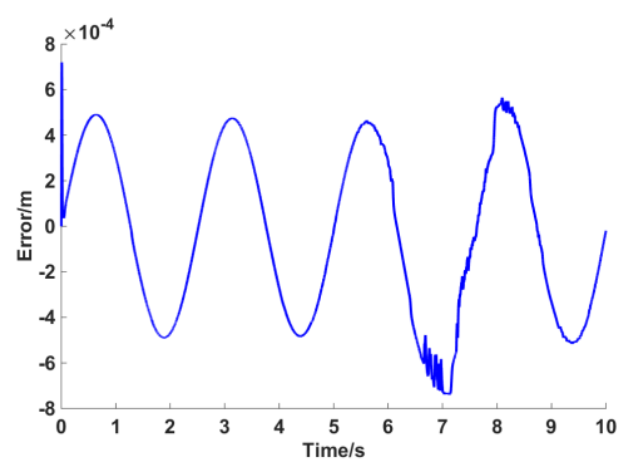

(b) $\mathrm{RC}$

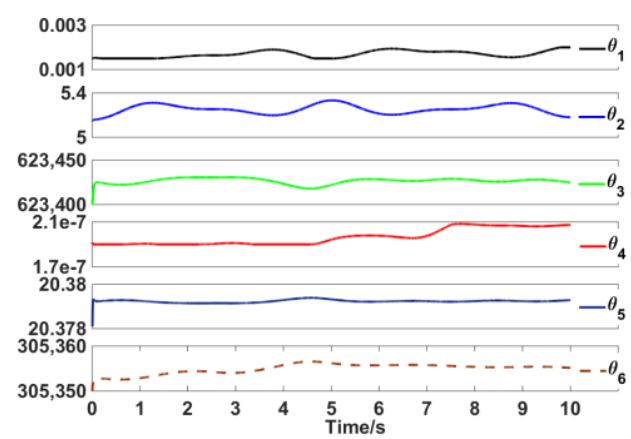

(d) Estimation of $\boldsymbol{\theta}$

Fig. 4: Comparative tracking performance of case 2. 


\section{Conclusion}

In this paper, a typical VCCS with uncertain parameters and unknown nonlinear disturbance was considered, and an adaptive robust dynamic surface control strategy was introduced to improve the control performance of the VCCS. The DSC technique was utilized to design the nonlinear controller of the considered VCCS, and the "explosion of complexity" problem of the traditional backstepping method was solved. Simultaneously, robust control method and projection-based parameter adaptation law were used to eliminate the effect of inevitable unknown nonlinear disturbance and parametric uncertainties in VCCS. The stability of the closed-loop system was analyzed by using Lyapunov method. At last, the comparative simulation results are obtained to verify the effectiveness of the proposed control strategy.

\section{Acknowledgements}

This research was supported by National Natural Foundation of China (51375304).

\section{References}

[1] H. Lin, Y. Yin and G. Salvendy, "Neural-network Based Fault Diagnosis of Hydraulic Forging Presses in China," International Journal of Production Research, vol. 33, no. 7, pp. 1939-1951, 2007.

[2] H. Yang, H. Shi and G. Gong, "Electro-hydraulic Proportional Control of Thrust System for shield Tunnelling Machine," Automation in Construction, vol. 18, no. 7, pp. 950-956, 2009.

[3] Y. Pi, X. Wang, "Trajectory Tracking Control of a 6-DOF Hydraulic Parallel Robot Manipulator with Uncertain Load Disturbances," Control Engineering Practice, vol. 19, no. 2, pp. 185-193, 2010.

[4] N. D'alfio, A. Morgando and A. Sorniotti, "Electro-hydraulic Brake Systems: Design and Test through Hardware-inthe-loop Simulation," Vehicle System Dynamics, vol. 44, no. 1, pp. 378-392, 2007.

[5] H. M. Chen, J. C. Renn and J. P. Su, "Sliding Mode Control with Varying Boundary Layers for an Electro-hydraulic Position Servo system," The International Journal of Advanced Manufacturing Technology, vol. 26, no. 1, pp. 117$123,2005$.

[6] M. Mihajlov, V. Nikolić and D. Antić, "Position Control of an Electro-hydraulic Servo System Using Sliding Mode Control Enhanced by Fuzzy PI Controller," Mechanical Engineering, vol. 1, no. 9, pp. 1217-1230, 2002.

[7] Y. He, J. Wang, W. Shen, "Indirect Adaptive Robust Dynamic Surface Control of Electro-hydraulic Fatigue Testing System with Huge Elastic Load," Systems and Control Engineering, vol. 230, no. 1, pp. 115-129, 2015.

[8] K. Sinthipsomboon, I. Hunsacharoonroj, J. Khedari, W. Pongaen, P. Pratumsuwan, "A hybrid of fuzzy and fuzzy self-tuning PID controller for servo electro-hydraulic system," 6th IEEE Conference on Industrial Electronics and Applications, Beijing, pp. 220-225, 2011.

[9] J. Han, "From PID to Active Disturbance Rejection Control," IEEE Transactions on Industrial Electronics, vol. 56, no. 3, pp. 900-906, 2009.

[10] W. M. Bessa, M. S. Dutra, E. Kreuzer, "Sliding Mode Control with Adaptive Fuzzy Dead Zone Compensation of an Electro-hydraulic Servo System," Journal of Intelligent \& Robotic Systems, vol. 58, no. 1, pp. 3-16, 2010.

[11] C. Guan and S. Pan, "Nonlinear Adaptive Robust Control of Single-Rod Electro-Hydraulic Actuator With Unknown Nonlinear Parameters," IEEE Transactions on Control Systems Technology, vol. 16, no. 3, pp. 434-445, 2008.

[12] X. Huang, X. Zhang and H. Lu, "Forwarding-based dynamic surface control for antagonistic actuated robots," IET Control Theory \& Applications, vol. 10, no. 15, pp. 1763-1770, 2016.

[13] W. Dong, J. A. Farrell, M. M. Polycarpou, V. Djapic and M. Sharma, "Command Filtered Adaptive Backstepping," IEEE Transactions on Control Systems Technology, vol. 20, no. 3, pp. 566-580, 2012.

[14] A. Mohanty and B. Yao, "Indirect Adaptive Robust Control of Hydraulic Manipulators With Accurate Parameter Estimates," IEEE Transactions on Control Systems Technology, vol. 19, no. 3, pp. 567-575, 2011.

[15] L. Xu and B. Yao, "Adaptive robust precision motion control of linear motors with negligible electrical dynamics: theory and experiments," IEEE/ASME Transactions on Mechatronics, vol. 6, no. 4, pp. 444-452, 2001.

[16] Z. Li, J. Chen, M. Gan, H. Fang and G. Zhang, "Adaptive robust dynamic surface control of DC torque motors with true parameter estimates," Proceedings of the 2010 American Control Conference, Baltimore, MD, pp. 3524-3529, 2010 . 